\title{
A Myelin Galactolipid, Sulfatide, Is Essential for Maintenance of Ion Channels on Myelinated Axon But Not Essential for Initial Cluster Formation
}

\author{
Tomoko Ishibashi, ${ }^{1,2,3}$ Jeffrey L. Dupree, ${ }^{4}$ Kazuhiro Ikenaka, ${ }^{1,3}$ Yukie Hirahara, ${ }^{5}$ Koichi Honke, ${ }^{6}$ Elior Peles, ${ }^{7}$ \\ Brian Popko, ${ }^{8}$ Kinuko Suzuki, ${ }^{9}$ Hitoo Nishino, ${ }^{10}$ and Hiroko Baba ${ }^{2}$ \\ ${ }^{1}$ Department of Physiological Sciences, The Graduate University for Advanced Studies, Okazaki 444-8585, Japan, \\ 2Department of Molecular Neurobiology, School of Pharmacy, Tokyo University of Pharmacy and Life Science, Hachioji \\ 192-0392, Japan, ${ }^{2}$ Laboratory of Neural Information, National Institute for Physiological Sciences, Okazaki National \\ Research Institutes, Okazaki 444-8585, Japan, 4Department of Pathology and Anatomy, Eastern Virginia Medical School, \\ Norfolk, Virginia 23507, ${ }^{5}$ Research Institute, Osaka Medical Center for Maternal and Child Health, Izumi 594-1101, Japan, \\ ${ }^{6}$ Department of Biochemistry, Osaka University Graduate School of Medicine, Suita 565-0871, Japan, '7Department of \\ Molecular Cell Biology, The Weizmann Institute of Science, Rehovot 76100, Israel, 8Neuroscience Center, Department of \\ Biochemistry and Biophysics, Program in Molecular Biology and Biotechnology and ${ }^{9}$ Department of Pathology and \\ Laboratory Medicine, University of North Carolina, Chapel Hill, North Carolina 27599, and 10Department of Physiology, \\ Nagoya City University Medical School, Nagoya 467-8601, Japan
}

Myelinated axons are divided into four distinct regions: the node of Ranvier, paranode, juxtaparanode, and internode, each of which is characterized by a specific set of axonal proteins. Voltage-gated $\mathrm{Na}^{+}$channels are clustered at high densities at the nodes, whereas shaker-type $\mathrm{K}^{+}$channels are concentrated at juxtaparanodal regions. These channels are separated by the paranodal regions, where septate-like junctions are formed between the axon and the myelinating glial cells. Although oligodendrocytes and myelin sheaths are believed to play an instructive role in the local differentiation of the axon to distinct domains, the molecular mechanisms involved are poorly understood. In the present study, we have examined the distribution of axonal components in mice incapable of synthesizing sulfatide by disruption of the galactosylceramide sulfotransferase gene. These mice displayed abnormal paranodal junctions in the CNS and PNS, whereas their compact myelin was pre- served. Immunohistochemical analysis demonstrated a decrease in $\mathrm{Na}^{+}$and $\mathrm{K}^{+}$channel clusters, altered nodal length, abnormal localization of $\mathrm{K}^{+}$channel clusters appearing primarily in the presumptive paranodal regions, and diffuse distribution of contactin-associated protein along the internode. Similar abnormalities have been reported previously in mice lacking both galactocerebroside and sulfatide. Interestingly, although no demyelination was observed, these channel clusters decreased markedly with age. The initial timing and the number of $\mathrm{Na}^{+}$channel clusters formed were normal during development. These results indicate a critical role for sulfatide in proper localization and maintenance of ion channels clusters, whereas they do not appear to be essential for initial cluster formation of $\mathrm{Na}^{+}$channels.

Key words: sulfatide; paranodal junction; myelin; node of Ranvier; $\mathrm{Na}^{+}$channel; $\mathrm{K}^{+}$channel
Myelinated axons are divided at the axonal surface into four discrete functional regions, including the internode, juxtaparanodal and paranodal regions, and the node of Ranvier (Peles and Salzer, 2000; Pedraza et al., 2001). The voltage-gated $\mathrm{Na}^{+}$channels are concentrated at the nodes of Ranvier, whereas shakertype $\mathrm{Kv} 1.1$ and $\mathrm{Kv} 1.2 \mathrm{~K}^{+}$channels are localized primarily at the juxtaparanodal regions. In addition, contactin-associated protein

Received Nov. 6, 2001; revised May 21, 2002; accepted May 21, 2002.

This work was supported by a Grant for Private Universities provided by the Ministry of Education, Culture, Sports, Science, and Technology, by the Promotion and Mutual Aid Corporation for Private Schools of Japan (both to H.B), and by Grants-in-Aid 10214204 and 13480270 from the Ministry of Education, Culture, Sports, Science, and Technology of Japan (K.I.). E.P. is an Incumbent of the Madeleine Haas Russell Career Development Chair. J.L.D. is supported by a grant from the National Multiple Sclerosis Society. This work was also supported by Grants NS27336 (B.P.) and NS24453 (K.S.) from the National Multiple Sclerosis Society.

Correspondence should be addressed to Dr. Hiroko Baba, Department of Molecular Neurobiology, School of Pharmacy, Tokyo University of Pharmacy and Life Science, 1432-1 Horinouchi, Hachioji, Tokyo 192-0392, Japan. E-mail: hirobaba@ps.toyaku.ac.jp.

Copyright (C) 2002 Society for Neuroscience $0270-6474 / 02 / 226507-08 \$ 15.00 / 0$
(Caspr) and its binding partner, contactin, form a complex at the paranodal junctions (Peles et al., 1997; Faivre-Sarrailh et al., 2000; Rios et al., 2000) and may physically separate these two channels by inhibiting lateral movement of these proteins. Oligodendrocytes and/or the myelin sheath are believed to play an instructive role in the discrete localizations of these channels; however, the molecular mechanisms that mediate these distributions remain largely undefined. During development, Caspr is first uniformly expressed on the surface of axons, and later, as myelination occurs, its distribution becomes restricted to the paranodal regions (Einheber et al., 1997; Rasband et al., 1999). Then, the ion channels form clusters sequentially. These observations suggest that Caspr may be a key axonal molecule in the formation of the paranodal junction, as well as the decision of channel localization. Support for this hypothesis has come from recent studies using mice lacking either myelin glycolipids (Dupree et al., 1999; Poliak et al., 2001), Caspr (Bhat et al., 2001), or contactin (Boyle et al., 2001), in which dissociation of the paranodal junctions and some abnormal distributions of ion channels are displayed. 
Mice generated with a disruption of the gene encoding UDPgalactose, ceramide galactosyltransferase (CGT), showed disruption of paranodal axo-glial junctions (Bosio et al., 1996; Coetzee et al., 1996; for review, see Popko, 2000). Immunohistochemical studies revealed abnormal distributions of $\mathrm{K}^{+}$channels and Caspr in the myelinated axons (Dupree et al., 1999; Poliak et al., 2001). However, because this mutant lacks two major glycolipids, galactocerebroside (GalC) and sulfatide, it is impossible to identify the independent function of these two lipids in the paranodal junction. More recently, mice incapable of synthesizing sulfatide but not GalC through disruption of galactosylceramide sulfotransferase (CST) gene have been generated (Honke et al., 2002). These mice are normal at the time of birth, develop neurological deficits after 6 weeks of life, and survive to $>1$ year of age. Thus the CST-deficient mouse is a useful tool for studying the function of sulfatide in myelin independent of that of GalC.

In the present study, we address the following two questions: First, does sulfatide have a unique function on the organization of axonal domain formation? Second, are the axonal channel clusters stable under the condition of such incomplete axo-glial junctions? To clarify these questions, the distributions of paranodal proteins as well as ion channels on the axons were examined in CST-deficient mice of different ages. Our findings suggest that sulfatide is not necessary for initial cluster formation but is an essential myelin component for the proper localization of axonal proteins as well as the maintenance of these proteins around the nodes.

\section{MATERIALS AND METHODS}

Antibodies. The polyclonal antibody against $\mathrm{Na}^{+}$channel was generated against a highly conserved 18 aa segment (TEEQKKYYNAMKKLGSKK) between homologous domains III and IV of the channel $\alpha$-subunit. A mouse monoclonal antibody, K58/35, against $\mathrm{Na}^{+}$channel (anti-pan $\mathrm{Na}^{+}$channel antibody) was purchased from Sigma (St. Louis, MO). The polyclonal and monoclonal antibodies against $\mathrm{K}^{+}$channel $\alpha$ - and $\beta$-subunits (Trimmer, 1991) and a mouse monoclonal antibody, 28/43, against amino acids 77-299 of human postsynaptic density (PSD)-95 were kindly provided by Dr. J. S. Trimmer (State University of New York, Stony Brook, NY) and were used at a dilution of 1:100. The polyclonal anti-Caspr antibody was used at a dilution of 1:2500 (Peles et al., 1997). A mouse monoclonal antibody against myelin basic protein (MBP) was purchased from Boehringer Mannheim (Mannheim, Germany). A rat monoclonal antibody (AA3) against the $\mathrm{C}$-terminal portion of myelin proteolipid protein (PLP) (Yamamura et al., 1991) was kindly provided by Dr. M. Lees (E. K. Shriver Center, Waltham, MA).

CST-deficient mice. The CST-deficient mice were created by gene targeting (Honke et al., 2002). The targeting construct was designed to replace the exon portions that encode for the transmembrane domain and the 5'-PAPS-binding motif in the CST gene with a neomycinresistance cassette. Genotypes of pups from intercross between heterozygous mice were determined by the PCR method using a specific primer set. The mouse line was maintained in the animal facility of the Osaka Medical Center for Maternal and Child Health (Osaka, Japan).

Immunohistochemistry. Optic nerves and cervical (C3-C5) spinal cords from homozygotes of CST-deficient mice as well as wild-type controls were fixed by transcardinal perfusion with $4 \%$ paraformaldehyde in $0.1 \mathrm{M}$ phosphate buffer (PB), $\mathrm{pH} 7.4$, and the tissue was cryoprotected with $30 \%$ sucrose in PBS, $\mathrm{pH} 7.4$, for $24 \mathrm{hr}$ at $4^{\circ} \mathrm{C}$. After embedding in optimal cutting temperature (OCT)-mounting medium (Miles, Elkhart, IN), the blocks were cut in $7-\mu \mathrm{m}$-thick sections. The sections were collected on 3-aminopropyltriethoxysilane (Sigma)-rubbed glass slides and allowed to air dry. After washing in PBS to remove OCT, the sections were permeabilized for $2 \mathrm{hr}$ in $0.1 \mathrm{M} \mathrm{PB}$, containing $0.3 \%$ Triton $\mathrm{X}-100$ and $10 \%$ goat serum, pH 7.4 (PBTGS). For double-labeling experiments, sections were incubated overnight at $4^{\circ} \mathrm{C}$ with primary antibodies diluted to appropriate concentration in PBTGS. Then the sections were thoroughly rinsed in PBS, followed by application of fluorescently labeled secondary antibodies for $40 \mathrm{~min}$ at room temperature (RT). Secondary antibodies used in this study were FITC-labeled goat anti-rabbit IgG (1:400; Cappel Lab- oratories, Aurora, $\mathrm{OH}$ ) and tetramethylrhodamine isothiocyanatelabeled goat anti-mouse IgG (1:400; Jackson ImmunoResearch, West Grove, PA). Finally, labeled cryosections were rinsed consecutively in PBTGS, $0.1 \mathrm{M} \mathrm{PB}$, and $0.05 \mathrm{M} \mathrm{PB}$ for $5 \mathrm{~min}$ each and mounted in Vectashield (Vector Laboratories, Burlingame, CA). Images were captured with a laser-scanning microscope LSM510 (Carl Zeiss, Oberkochen, Germany). Digitized images were transferred to a laboratory computer for later analysis using Image Pro (Media Cybernetics, Silver Spring, MD). In quantification of ion channel clusters and cluster lengths of $\mathrm{Na}^{+}$channel, a focal region of immunofluorescence was considered to represent a cluster (aggregate) of $\mathrm{Na}^{+}$or $\mathrm{K}^{+}$channels if it clearly stood out from background label, and the fluorescence intensity was $\geq 150$ using LSM510 software (Carl Zeiss). Based on the criteria mentioned above, the cluster numbers of ion channels were counted, and the length of each $\mathrm{Na}^{+}$channel cluster was measured by digital linear gauges using LSM510 software. The clusters with lower intensities $(<150)$ were also counted. Gauge outputs were transferred to a laboratory computer, and at least two sections of nerve from each animal were analyzed.

Electron microscopic analysis. CST-deficient and littermate wild-type mice $(14,29$, and 36 weeks of age) were intracardially perfused through the left ventricle with a fixation solution consisting of $4 \%$ paraformaldehyde and $2.5 \%$ glutaraldehyde in $0.1 \mathrm{M}$ Millonigs buffer, $\mathrm{pH}$ 7.3. The perfusions were followed by a 2 week incubation in the same fixative solution at $4^{\circ} \mathrm{C}$. Tissue samples from spinal cord and optic nerve were collected and processed for transmission electron microscopic analysis using standard techniques. Briefly, the optic nerves were sectioned 0.75 $\mathrm{cm}$ anterior to the optic chiasm and the spinal cord images were obtained from the ventral columns from level $\mathrm{C} 3$. The tissues were postfixed in $1 \%$ osmium tetroxide, dehydrated in acetone, and embedded in epoxy resin. Ultrathin sections were stained with uranyl acetate and lead citrate and then analyzed using a Leo 910 transmission electron microscope (Leo Electron Microscopy Ltd., Cambridge, UK).

Western blot analysis. Preparation of mouse total brain and immunoblotting were performed as described previously (Peles et al., 1995). Myelin was purified and extracted as described previously (Huber et al., 1994; Kim et al., 1995) based on the procedures of Norton and Poduslo (1973). Protein concentrations of the lysates were determined by BioRad Protein Assay (Bio-Rad Laboratories, Hercules, CA). The proteins $(5-50 \mu \mathrm{g})$ were analyzed by either $5,7.5$, or $15 \%$ SDS-PAGE and electroblotted to Hybond-P transfer membrane (Amersham Pharmacia, Buckinghamshire, UK). The blots were blocked for $1 \mathrm{hr}$ in $5 \%$ nonfat milk in $10 \mathrm{~mm}$ Tris- $\mathrm{HCl}, 100 \mathrm{~mm} \mathrm{NaCl}$, and $0.1 \%$ Tween 20, pH 7.5 (blocking buffer), at RT and probed with antibodies specific for antiCaspr (1:3000), anti-mouse monoclonal $\mathrm{Na}^{+}$channel antibody, K58/35 (1:1000), anti-MBP (1:3000), and anti-PLP (1:1000) diluted in blocking buffer for $1 \mathrm{hr}$ at RT. They were washed and exposed to goat anti-rabbit, mouse, and rat IgG conjugated by horseradish peroxidase diluted in blocking buffer for $30 \mathrm{~min}$ at RT. The conjugates were detected using the ECL system (Amersham Pharmacia), according to the manufacturer's instructions.

\section{RESULTS}

\section{CST-deficient mice exhibit a histological phenotype similar to CGT-deficient mice}

The CST-deficient mice, which lacked sulfatide but not GalC, exhibited paranodal abnormalities that were similar to CGTdeficient mice by electron microscopic study (Honke et al., 2002). To understand the function of myelin sulfatide on the characteristic domain formation of the axons, we first analyzed the distribution of the voltage-gated $\mathrm{Na}^{+}$and $\mathrm{K}^{+}$channels on the CNS (optic nerve and spinal cord) sections from the CST-deficient mice. In the control CNS axons, the $\mathrm{Na}^{+}$channels were concentrated at the nodes of Ranvier, and the $\mathrm{K}^{+}$channels were localized in the juxtaparanodal regions, which were adjacent to the paranodal regions as described previously (Fig. 1a). In contrast, the localization and form of both channels were altered on the CNS axons from the CST-deficient mice (Fig. 1b-e). In mutant mice, although $\mathrm{Na}^{+}$channels were concentrated in the small regions that were presumptive nodes of Ranvier, the lengths of the $\mathrm{Na}^{+}$channel clusters were occasionally longer than in the 

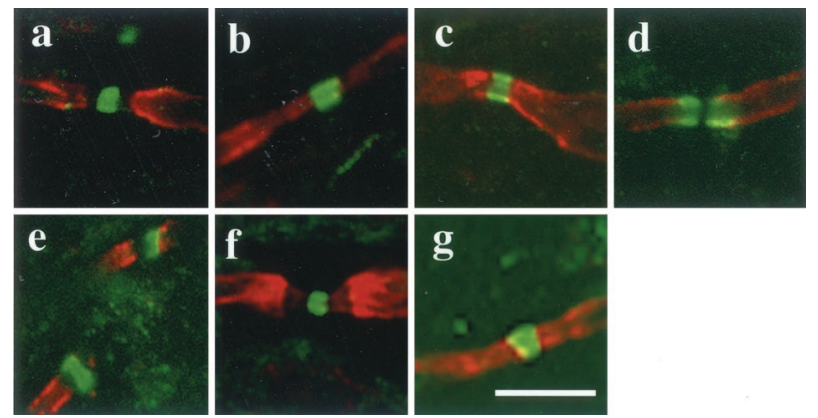

Figure 1. Alteration of ion channel clusters in CST-deficient mice. Double immunostaining of $\mathrm{Na}^{+}$and $\mathrm{K}^{+}$channels was performed in the CNS and PNS axons. $a$, In the spinal cord of wild-type mice, $\mathrm{Na}^{+}$ channels ( green) were restricted to the nodes of Ranvier, whereas the $\mathrm{K}^{+}$ channels (red) were concentrated in the juxtaparanodes. $b-e$, In contrast to the spinal cord of CST-deficient mice, the $\mathrm{K}^{+}$channels were aberrantly localized to the paranodes $(b, c, d)$. The $\mathrm{Na}^{+}$channels formed clusters in the nodes; however, the domains were longer $(b)$ in comparison with wild type $(a) . d$, The binary form of $\mathrm{Na}^{+}$channel clusters is frequently observed. $e$, Heminode formation that labeled just one side of the juxtaparanode or paranode with the Kv1.1 antibody was also observed. $f$, In the spinal root of wild-type mouse, $\mathrm{Na}^{+}$channels ( green) were restricted to the nodes of Ranvier, whereas the $\mathrm{K}^{+}$channels (red) were concentrated in the juxtaparanodes. $g$, In contrast, in the spinal root of this mutant mouse, the $\mathrm{K}^{+}$channel clusters were aberrantly localized to the paranode. Scale bar, $5 \mu \mathrm{m}$.

wild-type mice (Fig. 1, compare $a$ and $b$ ). We measured the nodal length in the optic nerves, which was indicated by the $\mathrm{Na}^{+}$ channel immunoreactivity. In 16-week-old littermate controls, the nodal lengths were nearly constant $(0.79 \pm 0.05 \mu \mathrm{m} ;>100$ nodes were examined from two mice). In contrast, in the optic nerve from the CST-deficient mice, the lengths were significantly longer in comparison with those of the control mice $(1.25 \pm 0.05 \mu \mathrm{m}$; $>75$ nodes were examined in each of two mice; $p<0.001$ by $t$ test). In addition, the binary appearance of $\mathrm{Na}^{+}$channel clusters was frequently observed in the mutant mice (Fig. $1 d$ ). In the optic nerves from the 10 -week-old mutant mice, $11 \%$ (25 of 246 clusters calibrating the use of two animals) of the $\mathrm{Na}^{+}$channel clusters were binary shaped, and $12 \%$ (31 of 245 clusters calibrating the use of two animals) were binary shaped in the 14-week-old mice. Such binary forms were never observed in wild-type mice of the same ages.

Conversely, $\mathrm{K}^{+}$channel distribution was significantly more affected in the CST-deficient mice. Although the $\mathrm{K}^{+}$channel antibody occasionally stained the paranodal regions in the wildtype mice, the intensity of the paranodal staining was significantly lower than that of the main juxtaparanodal clusters (Fig. 1a). In the mutant mice, in contrast, the $\mathrm{K}^{+}$channel clusters frequently accumulated in the region adjacent to the $\mathrm{Na}^{+}$channel cluster, in presumptive paranodal regions, and in the juxtaparanodal regions (Fig. $1 b-d$ ). As shown in Figure $1 c$, the intensity of the $\mathrm{K}^{+}$ channel antibody staining in some of the presumptive paranodes was even brighter than the staining in the juxtaparanodal regions. Heminodal formation that labeled just one side of the juxtaparanode or presumptive paranode with the antibody against Kv1.1 $\mathrm{K}^{+}$channel $\alpha$ subunit was also observed in the mutant mice (Fig. 1e). The staining patterns using the antibodies against other $\mathrm{K}^{+}$ channel subunits, $\mathrm{Kv} 1.2$ and $\mathrm{Kv} \beta 2$, revealed the same results (data not shown). These results were similar to the findings in the CGT-deficient mice in the CNS (Dupree et al., 1999).

We then examined whether the localizations of the ion channels in the PNS were affected to a similar extent as those in the
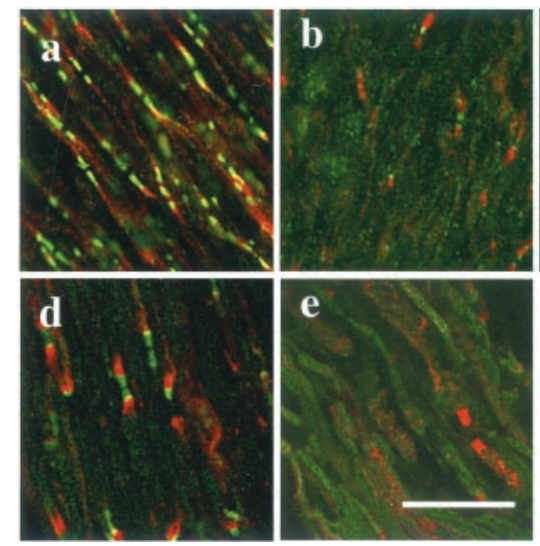

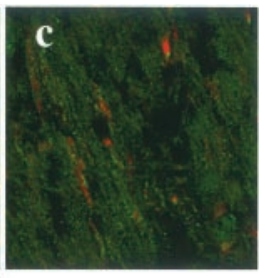

f

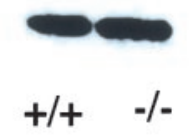

Figure 2. CST-deficient mice revealed severely disrupted Caspr clustering both in the CNS and in the PNS. $a$, $d$, In 6-week-old wild-type mice, Caspr (green) was highly concentrated in the paranodal regions of the optic nerve $(a)$ and in the paranodal region of the spinal root $(d) . b(6$ weeks), $c$ (10 weeks), $e$ (6 weeks), In contrast, CST-deficient mice exhibited a more diffuse labeling pattern. The number of $\mathrm{K}^{+}$channel clusters is markedly decreased in the mutant optic nerve as well as in the spinal root. $f$, Western blot analysis of brain homogenate revealed no differences in the amount of Caspr protein between wild-type $(+/+)$ and mutant $(-/-)$ mice in the CNS axons at 18 weeks of age. Scale bar, $10 \mu \mathrm{m}$.

CNS. The characteristic channel distribution was observed in the wild-type spinal root, as shown in Figure $1 f$, which was the same as in the spinal cord (Fig. 1a). However, the $\mathrm{K}^{+}$channels accumulated markedly in the presumptive paranodal as well as the juxtaparanodal regions (Fig. $1 g$ ), and the stained area occasionally overlapped with those of $\mathrm{Na}^{+}$channels in the mutant spinal root. These changes were quite similar to those in the mutant CNS (Fig. $1 b-d$ ). Thus, sulfatide seems to be a myelin component that is necessary for the proper domain formation of PNS as well as CNS axons.

Because disruption of the paranodal axo-glial junction was frequently observed by electron microscopic studies in CSTdeficient mice (Honke et al., 2002), we then analyzed the distribution of the axonal adhesion molecule Caspr. This protein is normally concentrated in the paranodal regions in the optic nerve as well as in the spinal root. Hence, double labeling with Caspr and $\mathrm{K}^{+}$channel antibodies revealed the paired patterns of these proteins next to the unlabeled node in the middle (Fig. $2 a, d$, respectively). On the contrary, this type of paired staining disappeared in the CST-deficient mice, and Caspr appeared to be diffusely distributed along the axons, as shown in Figure $2 b, c$ (optic nerve) and Figure $2 e$ (spinal root). In 6-week-old mice, although the small numbers of Caspr clusters were observed adjacent to some of the $\mathrm{K}^{+}$channel clusters, most of these channel clusters were not adjacent to Caspr (Fig. 2b). In 10-weekold mice, Caspr clusters were hardly recognized, and a diffuse staining pattern was observed throughout the axons in the CSTdeficient mice (Fig. 2c). This protein was diffusely distributed throughout the axolemma in the spinal root of the 6-week-old mutant mice as well (Fig. 2e). Western blot analysis of brain revealed no differences in the protein levels of Caspr between the CST-deficient and wild-type mice (Fig. 2f), indicating that the diminished immunoreactivity both in the CNS and in the PNS axons was, in fact, a result of dispersed distribution of this protein in the entire internode. These localization changes of axonal proteins were also observed in the CGT-deficient mice. Together, these results indicate that the lack of sulfatide is responsible for 

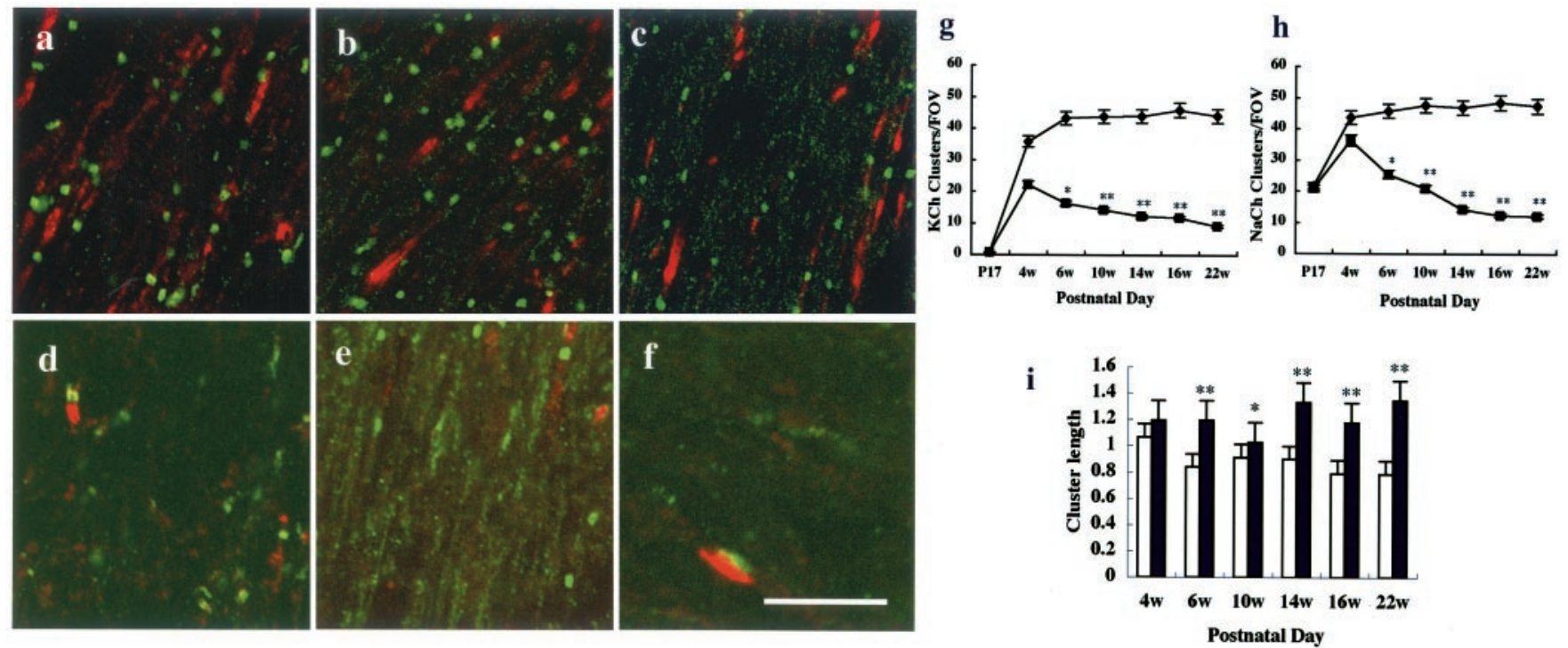

Figure 3. The ion channel clusters were reduced with age in CST-deficient mice. The optic nerves from 4-week-old $(a, d)$, 10-week-old $(b, e)$, and 22-week-old $(c, f)$ animals were double stained by $\mathrm{Na}^{+}$(green) and $\mathrm{K}^{+}($red $)$channel antibodies. In wild-type mice $(a-c)$, the numbers of $\mathrm{Na}^{+}$and $\mathrm{K}^{+}$ channel clusters were maintained without any changes throughout all ages. In the mutants $(d-f)$, both types of ion channel clusters were reduced, and irregular forms $(f)$ became prominent with age. Scale bar, $10 \mu \mathrm{m} . g$, $h$, Numbers of $\mathrm{K}^{+}$channel $(g)$ and $\mathrm{Na}^{+}$channel $(h)$ clusters per field of view in the optic nerves of wild-type $(\bullet)$ and mutant mice $(\square)$ plotted at different ages. An average of 12 FOVs was analyzed at each age. Single and double asterisks indicate $p<0.01$ and $p<0.05$, respectively. Wherever statistics are used, results are given as mean $\pm \mathrm{SD}$. $i$, The nodal lengths in the optic nerves of wild-type mice (white bar) and mutant mice (black bar), which were revealed by $\mathrm{Na}^{+}$channel immunoreactivity. Error bars indicate SD. Single and double asterisks indicate $p<0.001$ and $p<0.05$, respectively. 4w, Four weeks; $6 w$, six weeks; $10 w, 10$ weeks; $14 w, 14$ weeks; $16 w, 16$ weeks; $22 w, 22$ weeks.

the abnormal distribution of axonal proteins in both CGT- and CST-deficient mice.

\section{The ion channel clusters were markedly reduced with aging in the CST-deficient mice}

Recently, several mutants with disruptions of paranodal junctions have been reported, such as CGT-deficient mice (Dupree et al., 1998a,b), Caspr-deficient mice (Bhat et al., 2001), and contactindeficient mice (Boyle et al., 2001). All of these mice exhibited basically the same abnormal distributions of axonal proteins around the node as we have presently described in the CSTdeficient mice. However, the long-term effects of paranodal disclosure on the localization of axonal proteins still remained uncertain. To determine whether these abnormal paranodes eventually affect the ion channel localizations, we counted the numbers of channel clusters that showed the same fluorescence intensities as those in wild type in the optic nerves from postnatal day 17 (P17) to 22-week-old mice. These numbers were represented per field of view $(\mathrm{FOV})\left(1 \mathrm{FOV}=73.1 \times 73.1 \mu \mathrm{m}^{2}\right)$. An average of the numbers in 12 FOVs was calculated from littermates of the same age (Fig. 3). In the wild-type mice, the numbers of the $\mathrm{K}^{+}$(Fig. 3g) and $\mathrm{Na}^{+}$(Fig. 3h) channel clusters rapidly increased until 6 weeks or 4 weeks of age, respectively. These numbers were maintained without any significant changes throughout all of the ages examined (Fig. 3a-c, graph with diamond shapes in $g$ and $h$ ). In contrast, although the numbers of both channel clusters were already significantly lower in the optic nerves of 4-week-old CST-deficient mice in comparison with those of wild type, these numbers kept decreasing constantly with age (Fig. $3 d-f$, graph with squares in $g$ and $h$ ). Ultimately, these clusters disappeared dramatically in the 22-week-old optic nerve, and only $8 \%$ of the $\mathrm{K}^{+}$channel or $12 \%$ of the $\mathrm{Na}^{+}$channel clusters were detected in comparison with those of the wild type (Fig. $3 g, h$ ). Moreover the remaining channel clusters at this age exhibited irregular forms, as shown in Figure $3 f$ (arrow). In addition, the changes in the nodal lengths demonstrated by the $\mathrm{Na}^{+}$channel immunoreactivities were examined in the optic nerves from mice of different ages (Fig. 3i). Each column shows the length of $\mathrm{Na}^{+}$channel clusters in 4- to 22-week-old mice. The nodal lengths in the littermate controls (Fig. 3i, white columns) were nearly constant through $6-22$ weeks of age $(0.79 \pm 0.05 \mu \mathrm{m}$; $>95$ nodes were examined in two mice of each age). In the CST-deficient mice, the lengths from $>150$ nodes in two mice of each age (Fig. 3i, black columns) were significantly longer in all of the ages in comparison with the littermate controls except for 4-week-old animals ( $p<0.05$ or $p<0.001$ by $t$ test).

Because not only the numbers of $\mathrm{Na}^{+}$channel clusters but also the nodal lengths indicated by the $\mathrm{Na}^{+}$channel immunoreactivities were changed significantly after 6 weeks of age, we examined how these changes progressed with age. The optic nerves from 6 to 36 weeks of age were immunostained with anti-Na ${ }^{+}$channel $\alpha$ subunit antibody, and all of the confocal images were taken under the same conditions (detector gain and amplifier offset levels). Fluorescence intensities of almost all of the clusters in wild-type mice (Fig. 4a) were $>150$ using LSM510 software (see details in Materials and Methods). In contrast, various shapes of $\mathrm{Na}^{+}$ channel clusters with low-fluorescence intensities became more and more prominent in the older CST-deficient mice (Fig. $4 b-e$ ). Figure $4 g-i$ shows representative clusters with abnormal shapes (Fig. 4g,h, asterisks) and low intensities (Fig. 4h,i, arrowheads) compared with those in wild type (Fig. $4 f$ ). Therefore, we classified the clusters into two groups depending on their fluorescence intensities and counted these two groups of clusters independently in each age in the optic nerves from 6 to 36 weeks of age per FOV $\left(1 \mathrm{FOV}=48.7 \times 48.7 \mu \mathrm{m}^{2}\right)($ Table 1$)$. Even then, the total cluster numbers were significantly less in old mutant mice. In addition, the clusters with long shapes, as shown in Figure 4i, were 

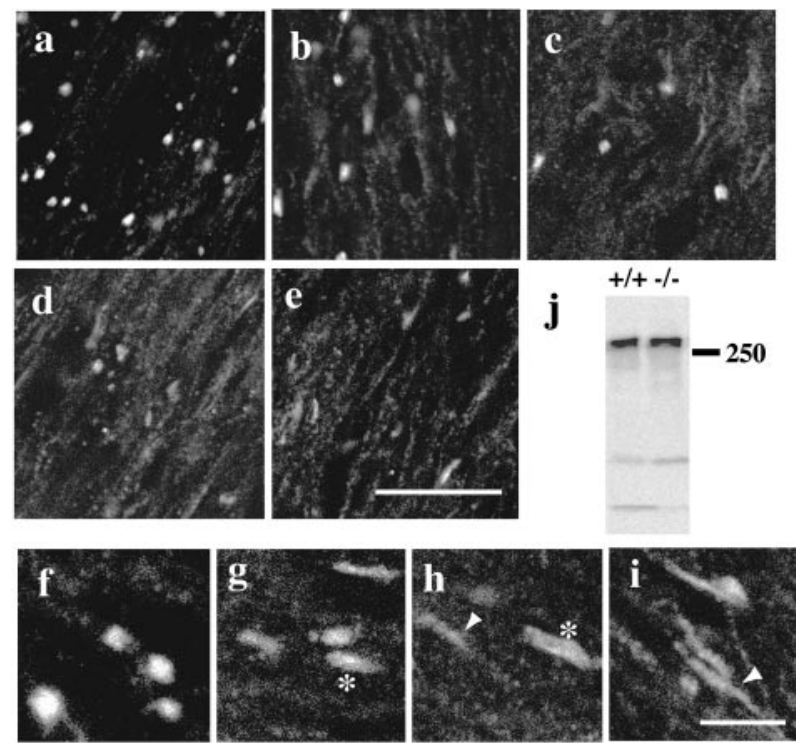

Figure 4. The changes of shapes and fluorescence intensities of $\mathrm{Na}^{+}$ channel clusters with age in CST-deficient mice. The optic nerves from wild-type mice $(a)$ and 6-week-old $(b), 16$-week-old $(c)$, 22-week-old $(d)$, and 36-week-old $(e)$ mutant mice were stained by $\mathrm{Na}^{+}$channel antibodies. In the mutant, the numbers of $\mathrm{Na}^{+}$channel clusters decreased significantly with age, and the clusters of longer shapes with lower intensities became more and more prominent in older mutant animals (compare $d$ or $e$ with $a$ ). $f$, Typical $\mathrm{Na}^{+}$channel clusters in wild-type mice whose intensity levels are $>150$ using LSM510 software (see Materials and Methods). $g-i$, The representative clusters with abnormal shapes ( $g$, $h, *)$ and intensities ( $h, i$, arrowheads) observed in the mutants. Scale bars: $a-e, 7 \mu \mathrm{m} ; f, g, 2 \mu \mathrm{m}$. $j$, An immunoblot of wild-type $(+/+)$ and mutant $(-/-)$ mice spinal cords at 36 weeks of age probed with anti-mouse $\mathrm{Na}^{+}$ channel antibody. The bands at $\sim 260 \mathrm{kDa}$ represent $\mathrm{Na}^{+}$channel $\alpha$ subunit. There were no differences in the amount of $\mathrm{Na}^{+}$channel protein between wild-type and mutant mice.

increased with age, and the relatively homogeneous staining along the axons was observed especially at 36 weeks of age (Fig. $4 e$ ), suggesting that $\mathrm{Na}^{+}$channels became distributed more diffusely.

The similar changes of $\mathrm{Na}^{+}$channel clusters were also observed in the spinal cord (data not shown). Western blot analysis of cervical spinal cord revealed no differences in the protein levels of $\mathrm{Na}^{+}$channels between the CST-deficient and wild-type mice (Fig. $4 j$ ). These findings suggested that sulfatide is necessary not only for the proper localization of channels but also for the maintenance of these channel clusters around the node. Furthermore, the CST-deficient mice showed a pronounced tremor and progressive ataxia from 6 weeks of age (Honke et al., 2002), when the loss of channel clusters became prominent. Thus, such maintenance of channel clusters may be critical for normal conduction.

\section{The CST-deficient mice do not exhibit apparent signs of demyelination}

Because the immunohistological abnormalities during aging in the CST-deficient mice were suggestive of demyelination, we examined the myelin membrane by immunostaining with the antibodies against two major myelin proteins, MBP and PLP/ DM20. No differences were observed in the staining pattern of MBP between the mutant and control optic nerves at 22 weeks of age (Fig. 5a,b), which is a different result from that observed in the demyelinating mutant mice generated by overexpression of PLP gene (Fig. 5c) (Kagawa et al., 1994). The PLP/DM20 stain- ing of the optic nerves also revealed no significant changes between the mutant and wild type (data not shown). Furthermore, each protein level in the myelin fraction of CNS was examined by Western blot analysis. As shown in Figure $5 d$,e, the intensities of the specific bands of MBP $(d)$ and PLP/DM20 (e) were unaltered between the mutants and the controls. Thus, even in 22-week-old mice, no evidence of demyelination was detectable by immunohistochemical as well as Western blot analysis. These results were confirmed by electron microscopic study. Cross-sectional analysis of optic nerves from 29-week-old wild-type (Fig. 5f) and CSTdeficient (Fig. 5g) mice revealed no difference in myelin stability. In addition, common indications of demyelination, such as myeloid figures and microglial activation, were not observed in the sulfatide-deficient mice at any of the ages analyzed. Spinal cord analysis revealed similar findings (data not shown). Together, these results demonstrated that the disappearance of the channel clusters during aging in this mutant was not attributable to demyelination but probably related to paranodal disruptions, although the axons were covered with normal-appearing compact myelin.

\section{The presence of Caspr is not a prerequisite to $\mathrm{Na}^{+}$ channel clustering at the nodes}

Because Caspr had been proposed to play an important role in channel clustering, we examined the localization of Caspr and $\mathrm{Na}^{+}$channel clusters carefully during development. At P17, during the period of rapid formation of the $\mathrm{Na}^{+}$channel clusters in the optic nerve (Fig. 6a), Caspr was distributed diff usely along the axons, and no clusters were observed in the CST-deficient mice in comparison with the littermates (Fig. 6b). In contrast to Caspr, no significant changes were observed in the number of $\mathrm{Na}^{+}$channel clusters of both mutant and wild-type mice $(23 \pm 4$ clusters per FOV in littermate control; $21 \pm 2$ clusters per FOV in the mutant mice; in both types of mice, $n=12$ from two animals each). Therefore, the clustering of Caspr may not be a prerequisite for the proper localization of the $\mathrm{Na}^{+}$channel at the presumptive node.

\section{The distribution of the PSD-95-related molecule is altered similarly to the distribution change of the $\mathrm{K}^{+}$channels}

Although it has not yet been fully characterized, PSD-95, which binds to the shaker-type $\mathrm{K}^{+}$channel $\alpha$-subunits (Kim et al., 1995), or a highly related protein colocalizes with the $\mathrm{K}^{+}$channels at the juxtaparanodal regions on myelinated axons (Baba et al., 1999). Therefore, we examined whether the localization of this protein was also altered in the CST-deficient mice. In contrast to wild-type mice, in which PSD-95-related protein formed clusters in the juxtaparanodal regions (Fig. $7 a$, red), this protein changed its localization to the paranodal regions adjacent to the $\mathrm{Na}^{+}$channel clusters in the CST-deficient mice (Fig. 7b). Double immunostaining using antibodies to PSD-95 and $\mathrm{K}^{+}$channel revealed the colocalization of these proteins in the paranodal region, suggesting that these two proteins may interact significantly even in the mutant mice (data not shown).

\section{DISCUSSION}

Sulfatide is an essential myelin component involved in the proper localization of axonal proteins through the axo-glial junction

Both electrical and histochemical experiments have revealed the importance of myelin GalC and/or sulfatide in proper paranodal formation (Dupree et al., 1998a,b, 1999). So which galactolipid is 
Table 1. Quantification of $\mathrm{Na}^{+}$channel clusters that classified into the fluorescence intensities

\begin{tabular}{|c|c|c|c|c|c|}
\hline \multirow[b]{2}{*}{$\begin{array}{l}\text { Fluorescence } \\
\text { intensity }\end{array}$} & \multirow{2}{*}{$\begin{array}{l}\text { Wild type } \\
6 \text { weeks } \\
(n=11)\end{array}$} & \multicolumn{4}{|c|}{ CST-deficient mice } \\
\hline & & $\begin{array}{l}6 \text { weeks } \\
(n=13)\end{array}$ & $\begin{array}{l}16 \text { weeks } \\
(n=11)\end{array}$ & $\begin{array}{l}22 \text { weeks } \\
(n=15)\end{array}$ & $\begin{array}{l}36 \text { weeks } \\
(n=14)\end{array}$ \\
\hline$\geq 150$ & $37 \pm 6$ & $17 \pm 4$ & $10 \pm 4$ & $5 \pm 3$ & $3 \pm 2$ \\
\hline$<150$ & $2 \pm 1$ & $13 \pm 5$ & $7 \pm 5$ & $7 \pm 3$ & $4 \pm 3$ \\
\hline
\end{tabular}

$\mathrm{The}^{+}{ }^{+}$channel clusters in the optic nerves were counted per FOV $\left(1 \mathrm{FOV}=48.7 \times 48.7 \mathrm{~mm}^{2}\right)$. The fluorescence intensity was classified as $\geq 150<150$ using LSM510 software.
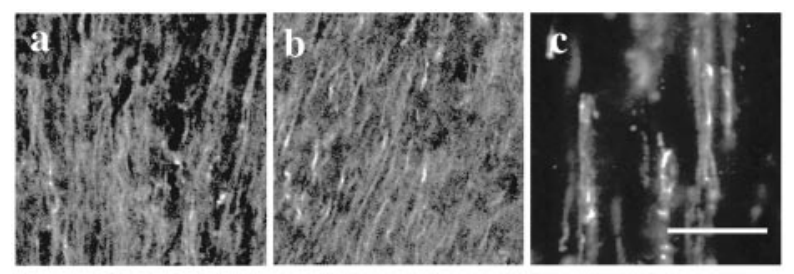

d

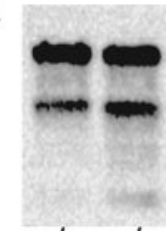

$+/+-/-$

f

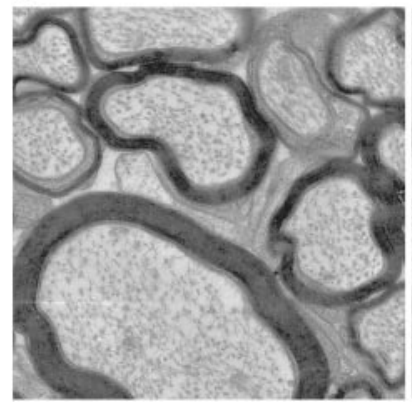

g

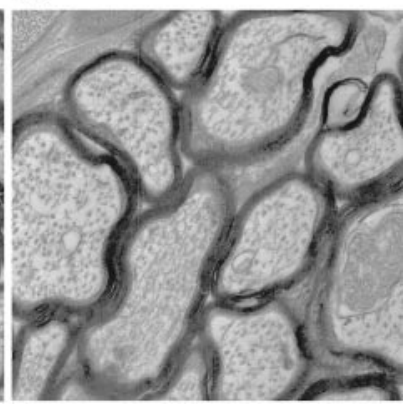

Figure 5. CST-deficient mice displayed no signs of demyelination. The staining pattern of $\operatorname{MBP}(a, b)$ exhibited no differences between wild-type (a) and CST-deficient $(b)$ mice in optic nerves at 22 weeks of age, whereas it is apparently different in demyelinating mutant mice generated by overexpression of PLP gene (Kagawa et al., 1996) at 28 weeks of age (c). Scale bar, $10 \mu \mathrm{m}$. Analysis of the membrane fraction of the brain from wild-type $(+/+)$ and mutant $(-/-)$ mice revealed no differences in the levels of MBP $(d)$ and PLP $(e)$ at 18 weeks of age. Electron microscopic analysis showed no signs of demyelination. Cross-sectional analysis of optic nerves from 29-week-old wild-type $(f)$ and CST-deficient $(g)$ mice revealed no difference in myelin stability. In addition, common indications of demyelination, such as myeloid figures and microglial activation, were not observed in the sulfatide-deficient mice at any of the ages analyzed. Spinal cord analysis revealed similar findings (data not shown).

essential for the formation of the axo-glial junction? The altered distributions of ion channels and Caspr both in the CNS and in the PNS of the CST-deficient mice are consistent with the observations from a previous report of CGT-deficient mice (Dupree et al., 1999). Despite the consistency in $\mathrm{Na}^{+}$channel cluster nodal location in both mutants, the lengths of the nodes indicated by $\mathrm{Na}^{+}$channel immunoreactivity were significantly longer in these mutants. The $\mathrm{K}^{+}$channel clusters were frequently observed in the paranodal regions rather than in the juxtaparanodal regions. Furthermore, Caspr clustering in the paranodal regions disap-
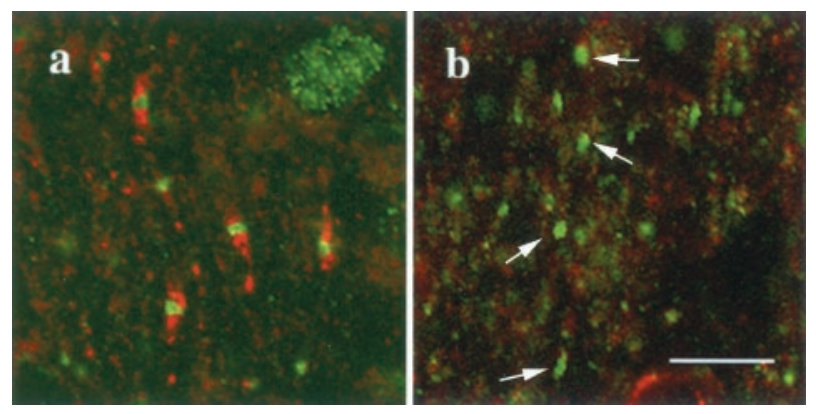

Figure 6. Optic nerves from CST-deficient mice exhibited severe disruptions in Caspr cluster formation during development (P17). Optic nerves from control littermates $(a)$ and CST-deficient mice $(b)$ were double labeled to indicate the localization of $\mathrm{Na}^{+}$channels ( green) and Caspr (red). Caspr clustering was hardly observed, and this protein was distributed diffusely along the axons in CST-deficient mice $(b)$. In contrast, $\mathrm{Na}^{+}$ channel cluster formation was comparable between mutants $(b$, arrows $)$ and control littermates. Scale bar, $10 \mu \mathrm{m}$.
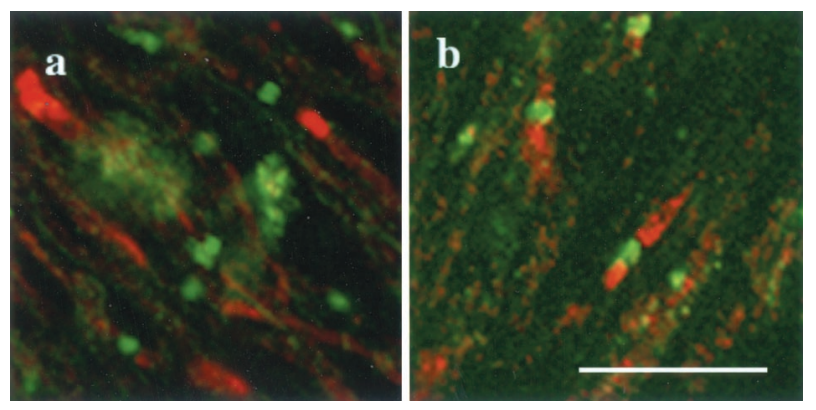

Figure 7. The distribution of PSD-95-related molecule is altered in CST-deficient mice. Optic nerves from control littermates $(a)$ and CSTdeficient mice $(b)$ were double labeled by the antibodies against $\mathrm{Na}^{+}$ channels (green) and PSD-95 (red). In the wild-type controls, the PSD95-related molecule formed clusters primarily in the juxtaparanodal regions, where the $\mathrm{K}^{+}$channels were usually localized. In contrast, in the mutants, the PSD-95-related molecule was concentrated in the paranodal regions rather than at the juxtaparanode and was sometimes distributed diffusely throughout the internode. Scale bar, $10 \mu \mathrm{m}$.

peared and became evenly distributed in the axolemma throughout the internode. Thus, all of these characteristic observations of the axonal proteins in CGT-deficient mice were well preserved in CST-deficient mice, suggesting that sulfatide, but not $\mathrm{GalC}$, is in fact a primary myelin component that determines the proper localization of axonal proteins via the paranodal axo-glial junctions.

Nevertheless, the clinical observations were distinct between the two mutants, and several additional histological changes were observed in CGT-deficient mice. For example, CGT-deficient mice exhibit a severe tremoring phenotype early on, within $14 \mathrm{~d}$, and die by $90 \mathrm{~d}$ of age. In contrast, the clinical phenotype of 
CST-deficient mice is milder, and these mice can survive to $>1$ year of age (Honke et al., 2002). Furthermore, CGT-deficient mice exhibit significant demyelination starting at $\sim 7$ weeks of age, and the CNS axons are almost completely devoid of myelin by 13 weeks of age (Dupree et al., 1998a). In contrast, according to our data, destruction of compact myelin does not occur in CST-deficient mice of the same age, and electron microscopic studies have demonstrated that it does not occur even by 36 weeks of age. These differences suggest that the two major glycolipids may function independently in myelin. For instance, sulfatide bears a negative charge and thus can bind electrostatically to any positively charged amino acid chain $\left(\mathrm{Arg}^{+}, \mathrm{His}^{+}\right.$, and $\left.\mathrm{Lys}^{+}\right)$on the surface of a protein (Vos et al., 1994). This kind of binding with galactolipid has been reported in several myelin proteins, such as MBP (for review, see Smith, 1992), myelin-oligodendrocyte basic proteins (Holz et al., 1996), and myelin and lymphocyte protein (Kim et al., 1995; Schaeren-Wiemers et al., 1995; Frank et al., 1998, 2000). Additionally, tenascin-R 160 and 180, two isoforms of an extracellular matrix molecule expressed in oligodendrocytes during myelination, have been reported to interact with sulfatide (Pesheva et al., 1997). Together, these results indicate that sulfatide may function differently on lipid-protein interactions. Hence, a defect in this lipid results in the disruption of junctional formation and in the abnormal localization of axonal ion channels. What, then, is the functional role of GalC in myelin? Based on the predominance of this glycolipid in myelin and on the significantly greater instability of myelin membranes in CGT-deficient mice in comparison with mice lacking only sulfatide, we surmise that GalC may be an essential myelin component to maintain structural integrity.

\section{Axo-glial interactions regulate axonal domain formation as well as its stability}

We have demonstrated that although no apparent demyelination was observed in CST-deficient mice, the numbers of clusters of both $\mathrm{K}^{+}$and $\mathrm{Na}^{+}$channels decreased significantly with age. Furthermore, Figure $4 j$ shows that $\mathrm{Na}^{+}$channels were not reduced at the protein level in this mutant, suggesting that the decrease in the clusters was attributable to the dispersed distribution of the channels throughout the axolemma. Our studies have provided the first evidence indicating that incomplete paranodal formation may eventually affect the stabilities of channel localization. The relative longevity ( $\geq 1$ year) of these mutant mice in comparison with other mutant mice with disruptions of paranodal junctions has allowed us to study whether sulfatide affects the stability of ion channel clusters.

Currently, three different mouse mutants are available for studying the molecular composition and functional roles of paranodal regions. Two of the mutants contain deficiencies in the axonal paranodal proteins Caspr or contactin, and the third contains a deficiency in the myelin glycolipids GalC and sulfatide. All mutants primarily exhibit disruptions of paranodal axo-glial junctions, as revealed by electron microscopic studies, and changes in axonal protein localization around the nodes. Caspr is distributed diffusely in the entire internode in the myelin mutants, whereas it is not found in the axons in both axonal mutants because this protein cannot be transported without contactin and stays in the neuronal cell bodies in the contactin knock-out mice. In each case, the $\mathrm{K}^{+}$channel clusters move to the presumptive paranodal regions, just next to the $\mathrm{Na}^{+}$channel clusters, but the densities of both channels in the internodes remain quite low. These changes are quite distinct from the changes observed in hypomyelinating (Baba et al., 1999; Rasband et al., 1999) and demyelinating mutants (Baba et al., 1999), in which both ion channels exhibit loss of specific localization and distribute diffusely in the axons. Thus, the presence of the compact myelin surrounding the axon can trigger channel clustering, although the complete axo-glial junction is necessary for the proper location of these clusters presumably because of the formation of a diffusion barrier between both channels, as reported recently (Poliak et al., 2001). It is also possible that the existence of paranodal loops of myelin in the absence of axolemma binding may be sufficient for the formation of channel clusters around the nodes.

However, at present it is still unclear whether the instability of axonal protein localization is specific for the CST-deficient mice or is a feature common to all mutants with paranodal abnormalities, because some other mutations result in lethality by postnatal day 20 , rendering them recalcitrant to observation with age.

\section{Caspr clustering is not essential for the initial determination of $\mathrm{Na}^{+}$channel clusters during development}

Based on previous reports, Caspr clustering is the initial event during CNS axonal formation that is strictly dependent on the maturation of the myelinated fibers, subsequent to which $\mathrm{Na}^{+}$ channel aggregation occurs, followed by the formation of $\mathrm{K}^{+}$ channel clusters (Rasband et al., 1999, 2000; Shrager, 2000). Proper development may not always necessitate the sequential occurrence of these events, because the present study using CSTdeficient mice clearly demonstrates that $\mathrm{Na}^{+}$channel clusters can be formed in appropriate regions at the correct time of development even in the absence of Caspr cluster formation in the beginning. It remains to be determined whether direct contact of the glial process is necessary to determine the localization of $\mathrm{Na}^{+}$ channel clusters. Nevertheless, the presence of Caspr protein in a specific place in the axolemma does not constitute the initial guidance step for ion channel clustering. Rather, a direct glial signal, either via a soluble factor (Kaplan et al., 1997) or through the mediation of actual contact, may determine future nodal location. This would be followed by the formation of $\mathrm{K}^{+}$channel clusters in the presence of compact myelin, the localization of which is contingent on the presence or absence of the axo-glial junction.

\section{How might sulfatide mediate interactions between myelinating glial cell and the axon?}

Recently, Bansal et al. (1999) suggested that sulfatide and/or GalC interact with external ligands and negatively regulate oligodendrocyte differentiation. So it is possible that sulfatide may directly bind to an axolemmal partner and mediate some signals through axo-glial association. However, the following hypothesis can be proposed to explain the dramatic alteration in the paranodal regions observed in CST-deficient mice. Namely, sulfatide may indirectly mediate axo-glial junctions by regulating intracellular protein trafficking in myelin-forming cells, and hence, myelin protein trafficking may be disrupted in CST-deficient mice. In support of this possibility, there has been evidence demonstrating that glycolipids actively select certain proteins for subcellular transport via detergent-insoluble, glycolipid-enriched complexes [digoxigenin (DIG)] (Ledesma et al., 1998). In many cell types, these lipid-dependent domains are usually enriched in glucocerebroside (Simons and Ikonen, 1997). However, the composition of myelin is atypical in many aspects. In comparison with other cell membranes, myelin contains a high amount of lipid (75-80\% of dry weight), with galactosphingolipids in particular contribut- 
ing $\sim 30 \%$ of the weight of total lipids (for review, see Vos et al., 1994). In addition, Kramer et al. (1997) have demonstrated that the galactolipid DIGs, isolated from maturing oligodendrocytes and the myelin sheath, contain the glycosyl phosphatidylinositollinked proteins known as neural cell adhesion molecule 120 and contactin. The protein composition of DIGs prepared from CGTdeficient mice was changed compared with that of normal brains (Bansal et al., 1999). Recently, neurofascin 155, an ankyrinbinding member of the L1 family, was identified as the first glial molecule enriched in paranodal regions (Tait et al., 2000). Poliak et al. (2001) reported that the disruption of clustering of this protein was observed in CGT-deficient mice. We also found that clustering of neurofascin 155 was hardly detected in the paranodal regions in the CST-deficient mice (T. Ishibashi and H. Baba, unpublished observation). Thus, sulfatide may collect such adhesion molecules in DIGs and transport them to form axo-glial junctions.

\section{REFERENCES}

Baba H, Akita H, Ishibashi T, Inoue Y, Nakahira K, Ikenaka K (1999) Completion of myelin compaction, but not the attachment of oligodendroglial processes triggers $\mathrm{K}+$ channel clustering. J Neurosci Res 58:752-764.

Bansal R, Winkler S, Bheddah S (1999) Negative regulation of oligodendrocyte differentiation by galactosphingolipids. J Neurosci 19:7913-7924.

Bhat MA, Rios JC, Lu Y, Garcia-Fresco GP, Ching W, Martine MS, Li J, Einheber S, Chesler M, Rosenbluth J, Salzer JL, Bellen HJ (2001) Axo-glial interactions and the domain organization of myelinated axons requires neurexin 4/Caspr/Paranodin. Neuron 30:369-383.

Bosio A, Binczek E, Stoffle W (1996) Fuctional breakdown of the lipid bilayer of the myelin membrane in central and peripheral nervous system by disrupted galactocerebroside synthesis. Proc Natl Acad Sci USA 12:13280-13285.

Boyle MET, Berglund EO, Murai KK, Weber L, Peles E, Ranscht B (2001) Contactin orchestrates assembly of septate-like junctions at the paranode in myelinated peripheral nerve. Neuron 30:385-397.

Coetzee T, Fujita N, Dupree J, Shi R, Bright A, Suzuki K, Popko B (1996) Myelination in the absence of galactocerebroside and sulfatide: normal structure and abnormal function and regional instability. Cell 86:209-219.

Dupree JL, Coetzee T, Blight A, Suzuki K, Popko B (1998a) Myelin galactolipids are essential for proper node of Ranvier formation in the CNS. J Neurosci 18:1642-1649.

Dupree JL, Coetzee T, Suzuki K, Popko B (1998b) Myelin abnormalities in mice deficient in galactocerebroside and sulfatide. J Neurocytol 27:649-659.

Dupree JL, Girault J-A, Popko B (1999) Axo-glial interactions regulate the localization of axonal paranodal proteins. J Cell Biol 147:1145-1151.

Einheber S, Zanazzi G, Ching W, Scherer S, Milner TA, Peles E, Salzer JL (1997) The axonal membrane protein Caspr, a homologue of neurexin IV, is a component of the septate-like paranodal junctions that assemble during myelination. J Cell Biol 139:1495-1506.

Faivre-Sarrailh C, Gauthier F, Denisenko-Nehrbass N, Bivic A, Rougon G, Girault JA (2000) The glycosylphosphatidyl inositol-anchored adhesion molecule F3/contaction is required for surface transport of paranodin/contactin-associated protein (caspr). J Cell Biol 149:491-501.

Frank M (2000) MAL, a proteolipid in glycosphingolipid enriched domains: functional implications in myelin and beyond. Prog Neurobiol 60:531-544.

Frank M, van der Haar ME, Schaeren-Wiemers N, Schwab ME (1998) rMAL is a glycosphingolipid-associated protein of myelin and apical membranes of epithelial cells in kidney and stomach. J Neurosci 18:4901-4913.

Holz A, Schaeren-Wiemers N, Schaefer C, Pott U, Colello RJ, Schwab ME (1996) Molecular and developmental characterization of novel cDNAs of the myelin-associated/oligodendrocytic basic protein. J Neurosci 16:467-477.

Honke K, Hirahara Y, Dupree J, Suzuki K, Popko B, Fukushima J,
Nagasawa T, Yoshida N, Wada Y, Taniguchi N (2002) Paranodal junction formation and spermatogenesis require sulfoglycolipids. Proc Natl Acad Sci USA 99:4227-4232.

Huber LA, Madison DL, Simons K, Pfeiffer SE (1994) Myelin membrane biologenesis by oligodendrocytes: developmental regulation of low-molecular weight GTP-binding proteins. FEBS Lett 347:273-278.

Kagawa T, Ikenaka K, Inoue Y, Kuriyama S, Tsuji T, Nakao J, Nakajima K, Aruga J, Okano H, Mikoshiba K (1994) Glial cell degeneration and hypomyelination caused by overexpression of myelin proteolipid protein gene. Neuron 13:427-442.

Kaplan MR, Meyer-Franke A, Lambert S, Bennet V, Duncan ID, Levinson SR, Barres BA (1997) Induction of sodium channel clustering by oligodendrocytes. Nature 136:724-728.

Kim T, Fiedler K, Madison DL, Krueger WH, Pfeiffer SE (1995) Cloning and characterization of MVP17: a developmentally regulated myelin protein in oligodendrocytes. J Neurosci Res 42:413-422.

Kramer EM, Koch T, Niehaus A, Trotter J (1997) Oligodendrocytes direct glycosyl phosphatidylinositol-anchored proteins to the myelin sheath in glycosphingolipid-rich complexes. J Biol Chem 272:8937-8945.

Ledesma MD, Simons K, Dotti CG (1998) Neuronal polarity: essential role of protein-lipid complexes in axonal sorting. Proc Natl Acad Sci USA 95:3966-3971.

Norton WT, Poduslo SE (1973) Myelination in rat brain: method of myelin isolation. J Neurochem 21:749-757.

Pedraza L, Huang JK, Colman DR (2001) Organizing principles of the axoglial apparatus. Neuron 30:335-344.

Peles E, Salzer JL (2000) Functional domains in myelinated axons. Curr Opin Neurobiol 10:558-565.

Peles E, Nativ M, Campbell PL, Sakurai T, Martinez R, Lev S, Clary DO, Schilling J, Barnea G, Plowman GD (1995) The carbonic anhydrase domain of receptor tyrosine phosphatase $\beta$ is a functional ligand for the axonal cell recognition molecule contactin. Cell 82:251-260.

Peles E, Nativ M, Lustig M, Grumet M, Schilling J, Martinez R, Plowman GD, Schlessinger J (1997) Identification of a novel contactinassociated transmembrane receptor with multiple domains implicated in protein-protein interactions. EMBO J 16:978-988.

Pesheva P, Gloor S, Schachner M, Probstmeier R (1997) Tenascin-R is an intrinsic autocrine factor for oligodendrocyte differentiation and promotes cell adhesion by a sulfatide-mediated mechanism. J Neurosci 17:4642-4651.

Poliak S, Gollan L, Salomon D, Berglund EO, Ohara R, Ranscht B, Peles E (2001) Localization of Caspr2 in myelinated nerves depends on axo-glia interactions and the generation of barriers along the axon. J Neurosci 21:7568-7575.

Popko B (2000) Myelin galactolipids: mediators of axon-glial interaction? Glia 29:149-153.

Rasband MN, Shrager P (2000) Ion channel sequestration in central nervous system axons. J Physiol (Lond) 525:63-73.

Rasband MN, Peles E, Trimmer JS, Levinson R, Lux SE, Shrager P (1999) Dependence of nodal sodium channel clustering of paranodal axoglial contact in the developing CNS. J Neurosci 19:7516-7528.

Rios JC, Melendez-Vasquez CV, Einheber S, Lustig M, Grumet M, Hemperly J, Peles E, Salzer JL (2000) Contactin-associated protein (Caspr) and contactin form a complex that is targeted to the paranodal junctions during myelination. J Neurosci 20:8354-8364.

Schaeren-Wiemers N, Valenzuela DM, Frank M, Schwab ME (1995) Characterization of a rat gene, rMAL, encoding a protein with four hydrophobic domains in central and peripheral myelin. J Neurosci 15:5753-5764.

Simons K, Ikonen E (1997) Functional rafts in cell membranes. Nature 387:569-572.

Smith R (1992) The basic protein of CNS myelin: its structure and ligand binding. J Neurochem 59:1589-1608.

Tait S, Gunn-Moore F, Collinson JM, Huang J, Lubetzki C, Pedraza L, Sherman DL, Colman DR, Brophy PJ (2000) An oligodendrocyte cell adhesion molecule at the site of assembly of the paranodal axo-glial junction. J Cell Biol 150:657-666.

Trimmer JS (1991) Immunological identification and characterization of a delayed rectifier $\mathrm{K}^{+}$channels polypeptide in rat brain. Proc Natl Acad Sci USA 88:10764-10768.

Vos JP, Lopes-Cardozo M, Gadella BM (1994) Metabolic and functional aspects of sulfogalactolipids. Biochim Biophys Acta 1211:125-149.

Yamamura T, Konola JT, Wekerle H, Lees MB (1991) Monoclonal antibodies against myelin proteolipid protein: identification and characterization of two major determinants. J Neurochem 57:1671-1680. 